Bull. Mater. Sci., Vol. 3, Number 4, December 1981, pp. 389-397. (C) Printed in India.

\title{
Dislocation network patterns in tungstenite single crystals
}

\author{
M K AGARWAL, K NAGI REDDY*, J V PATEL $\dagger$ and \\ N G PATEL \\ Department of Physics, Sardar Patel University, Vallabh Vidyanagar 388 120, India \\ *Department of Physics, KSRM College of Engineering, Cuddapah, India. \\ $\dagger$ Department of Physics, Shree Jayendrapuri Arts and Science College, Broach 392002, \\ India.
}

MS received 10 December 1980; revised 27 January 1981

\begin{abstract}
TEM studies of tungstenite crystals grown by sublimation method have been investigated. Different types of dislocation network patterns were observed. The observed network patterns in bright field are compared with weak beam dark field pictures and the results are discussed.
\end{abstract}

Keywords. Dislocation network; tungstenite;

\section{Introduction}

Tungstenite $\left(\mathrm{WS}_{2}\right)$ is an isomorph of molybdenite. It belongs to a class of group VI dichalcogenides having $\mathrm{C}_{7}$ type crystal structure, which possesses a layered scructure. These $\mathrm{MX}_{2}(\mathrm{M}=\mathrm{W}$ or $\mathrm{Mo}, \mathrm{X}=\mathrm{S}, \mathrm{Se}$, or $\mathrm{Te})$ compounds form a structurally and chemically well defined family. The basic structure of loosely coupled X-M-X sheets makes such materials extremely interesting in that, within a layer, the bonds are strong, while between adjacent layers they are remarkably weak. As a result the crystals have an easy basal cleavage.

It has been reported (Amelinckx and Delavignette, 1962 and Basinski et al 1963) that the properties of materials which crystallize with a layer structure are influenced considerably by dislocations lying in the basal planes. Transmission electron microscopy is a well established technique for studying basal dislocations in crystals. Amelinckx and Delavignette (1962) have carried out a detailed transmission electron microscopic (TEM) study of $\mathrm{MoS}_{2}$ using bright field and dark field techniques. Recently, Agarwal et al (1979b) have carried out such studies on $\mathrm{MoSe}_{2}$ crystals. To our knowledge no (TEM) studies of $\mathrm{WS}_{2}$ have so far been reported. This may be because of its rare occurrence in nature. We have been able to grow them in considerably large size by a sublimation method (Agarwal et al 1979a). The present paper deals with the TEM studies on these crystals using the weak beam dark field technique.

It was shown by Cockayne et al (1969) that considerable improvements in the resolution of the structure of lattice defects can be obtained from dark field electron micrographs taken in weak diffracted beams. This can be done because the weak beam intensity can be considerably increased only very close to the defect structure where sufficiently large local tilts of the crystal occur to bring the weak beam nearer to the Bragg position. As a result dislocations appear as very narrow bright lines against a low intensity back-ground. This means that much more details can be 
resolved in weak beam images than in normal bright field or dark field images taken close to Bragg reflecting conditions and accurate measurements of dislocation positions can be made.

\section{Experimental}

Specimens for TEM studies were prepared by repeated cleavage (Agarwal and Babu Joseph, 1974). These specimens were examined by electron optical microscope (Philips EM 400). For taking photographs using the weak beam techmique first of all a strong two beam dark field condition is set up for $(\bar{h}, \bar{k}, \bar{l})$ using beam tilt settings $X$ and $Y$ and focussing and adjustments are carried out on this image. The weak beam condition for the systematic $(\bar{h}, \bar{k}, \bar{l})$ reflections can then be achieved immediately by selecting the opposite deflection fields settings $-X,-Y$; photographs can then be taken blind if necessary.

\section{Results and discussion}

The networks of dislocations are in fact twist boundaries with a $c$-rotation axis. They result from the orientation of two families of basal dislocations, with a different Burgers vector. If the two sets are in the same lattice plane, the result is a network of extended and contracted nodes as shown schematically in figure 1a. As the mesh size decreases the curvature of the sides of the stacking fault triangles becomes smaller and smaller and finally a network consisting of triangular meshes is formed. Alternate triangles are faulted. This is shown schematically in figure 1b. An observed example is shown in figure 2, which shows a family of two fold ribbons crossing the network and in addition to this the stacking fault triangles are also seen. Whenever
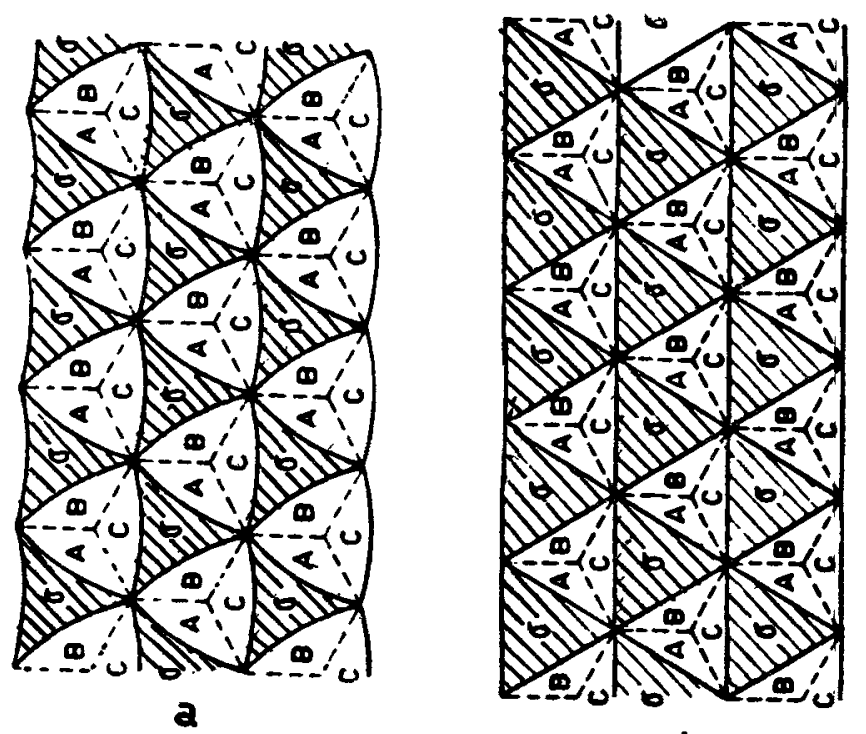

b

Figures 1a and 1b. Schematic diagram of extended and contracted nodes. 

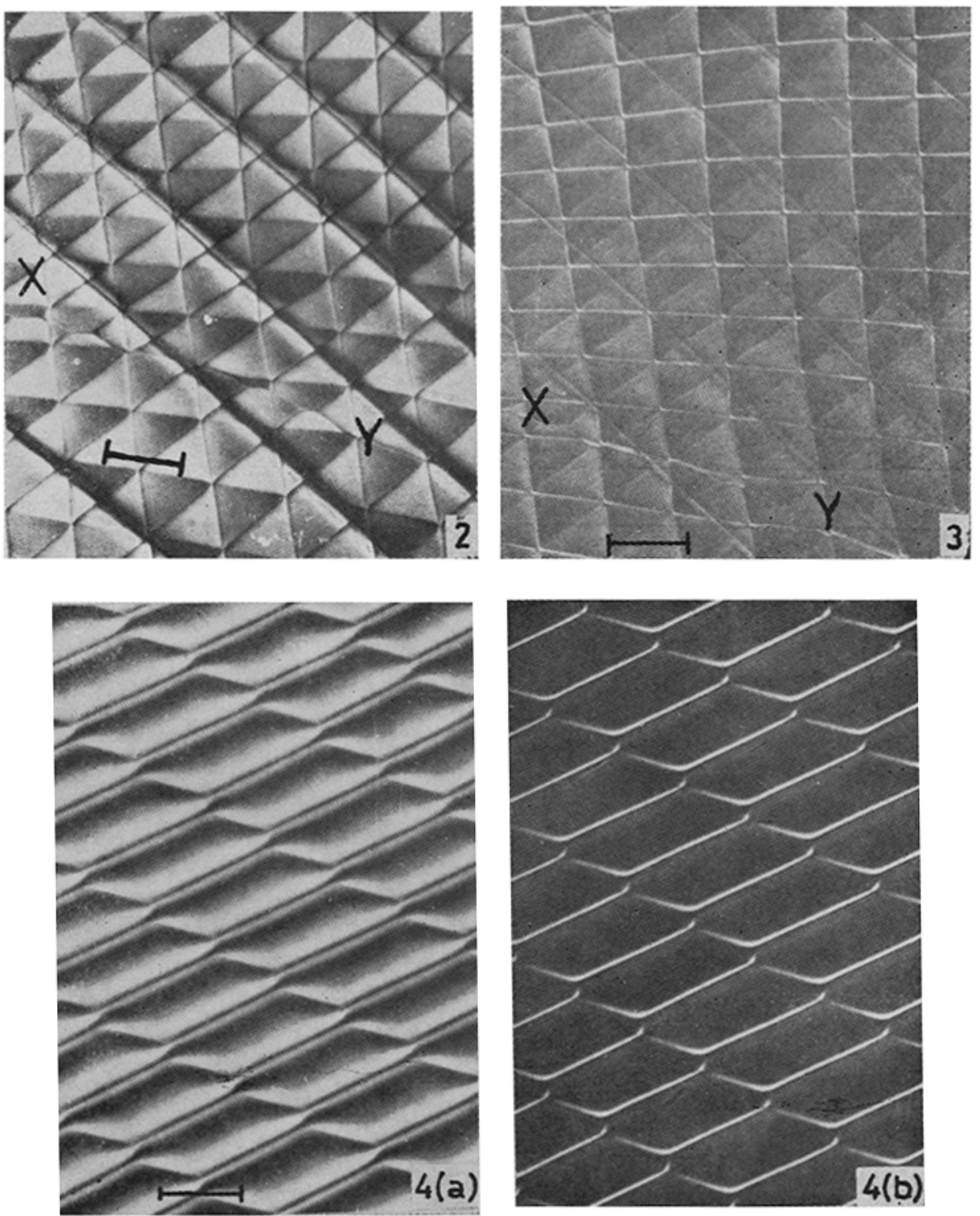

Figure 2. Bright field picture of extended and contracted nodes. Bar $=35 \mu \mathrm{m}$. Figure 3. Weak beam dark field picture of Fig. 2. Bar $=35 \mu \mathrm{m}$.

Figure 4a. Bright field picture of network pattern. Bar $=14 \mu \mathrm{m}$.

Figure 4b. Weak beam picture of figure 4a. $B a r=14 \mu \mathrm{m}$. 

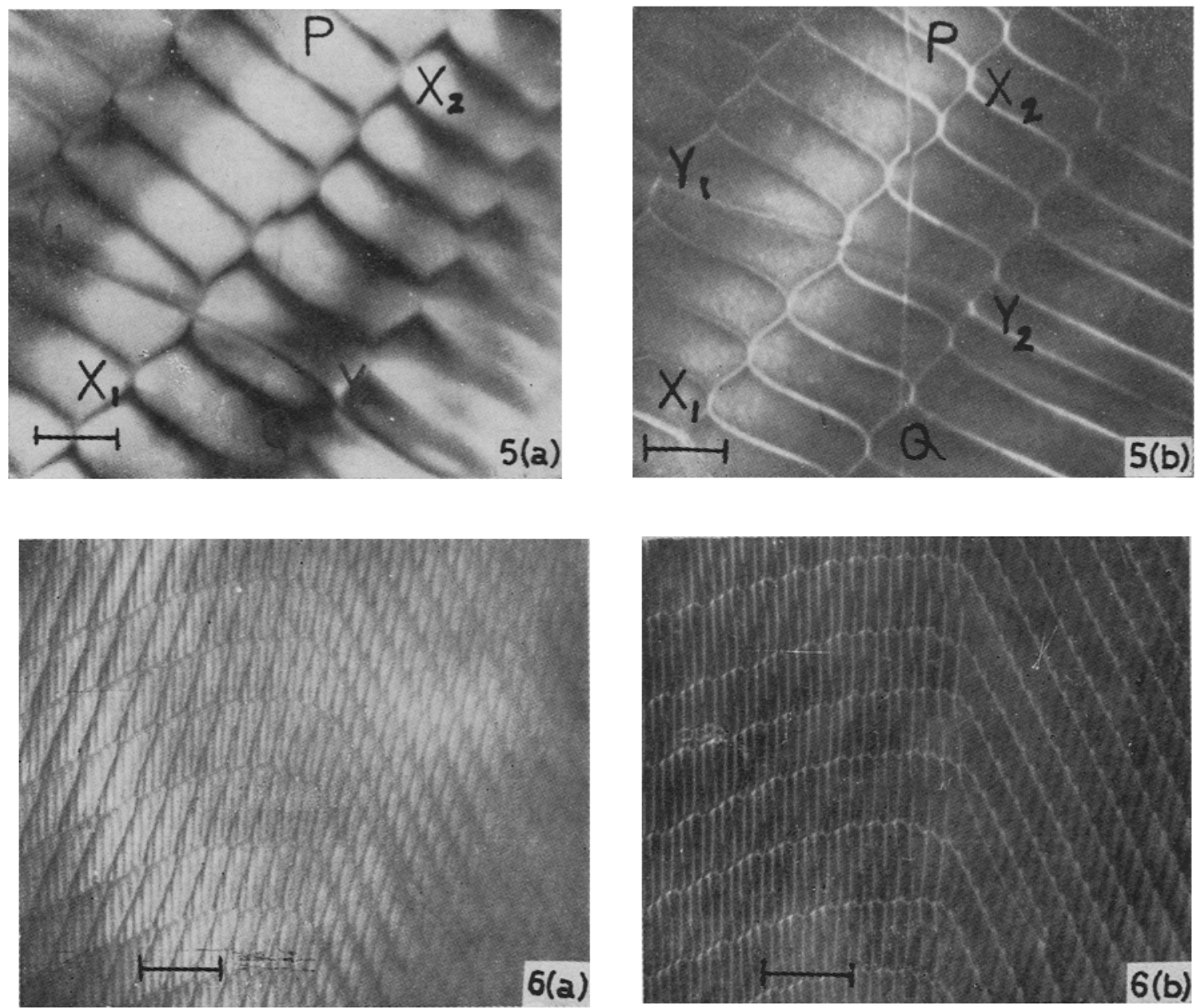

Figure 5a. Bright field picture of a network pattern formed due to a family of ribbons. Bar $=16 \mu \mathrm{m}$.

Figure 5b. Weak beam picture of figure 5a. Bar $=16 \mu \mathrm{m}$.

Figure 6a. Network pattern formed due to the three family of ribbons. Bar $=27 \mu \mathrm{m}$

Figure 6b. Weak beam picture of figure $6 a$. Bar $=27 \mu \mathrm{m}$. 

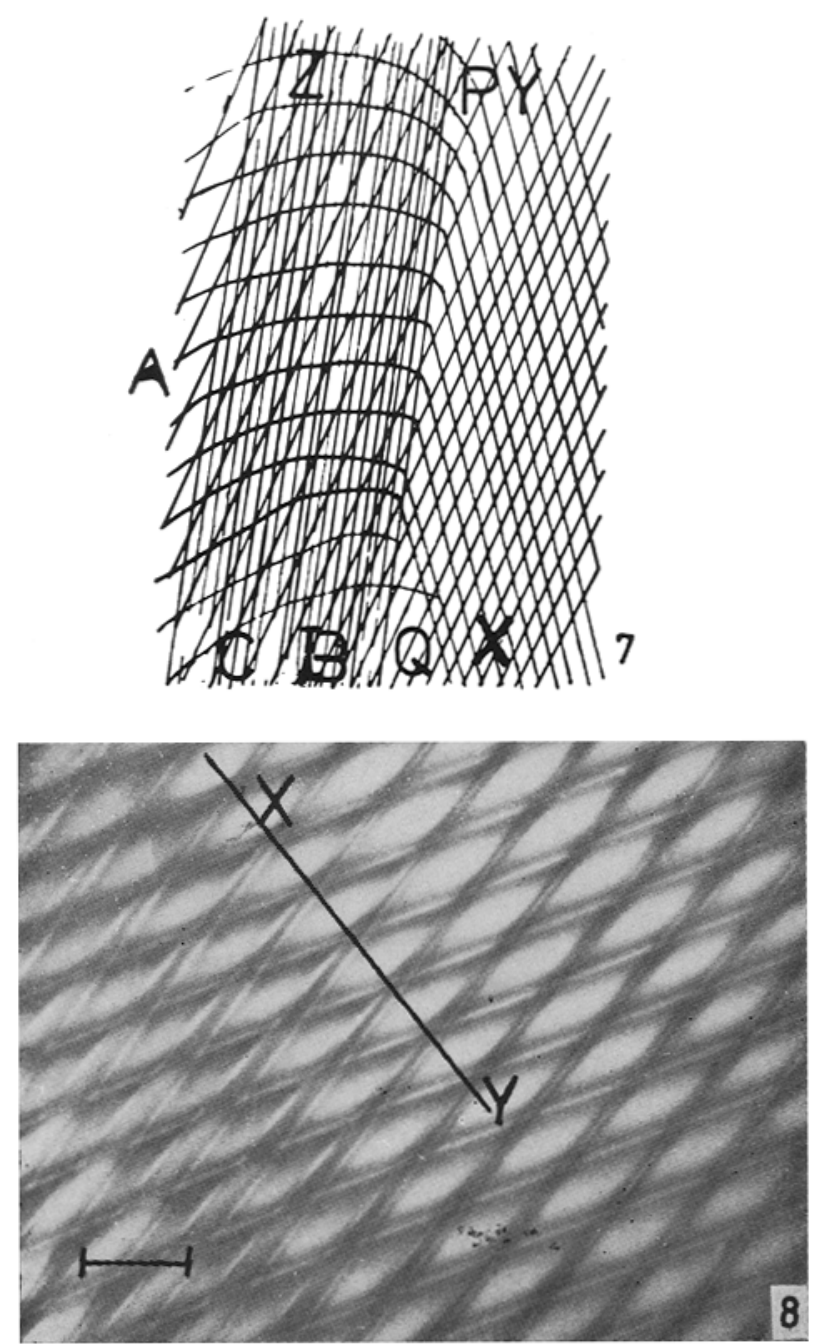

Figure 7. Schematic diagram showing a network pattern by three sets of ribbons.

Figure 8. Network pattern formed due to two sets of two fold ribbons. Bar $=7 \mu \mathrm{m}$. 

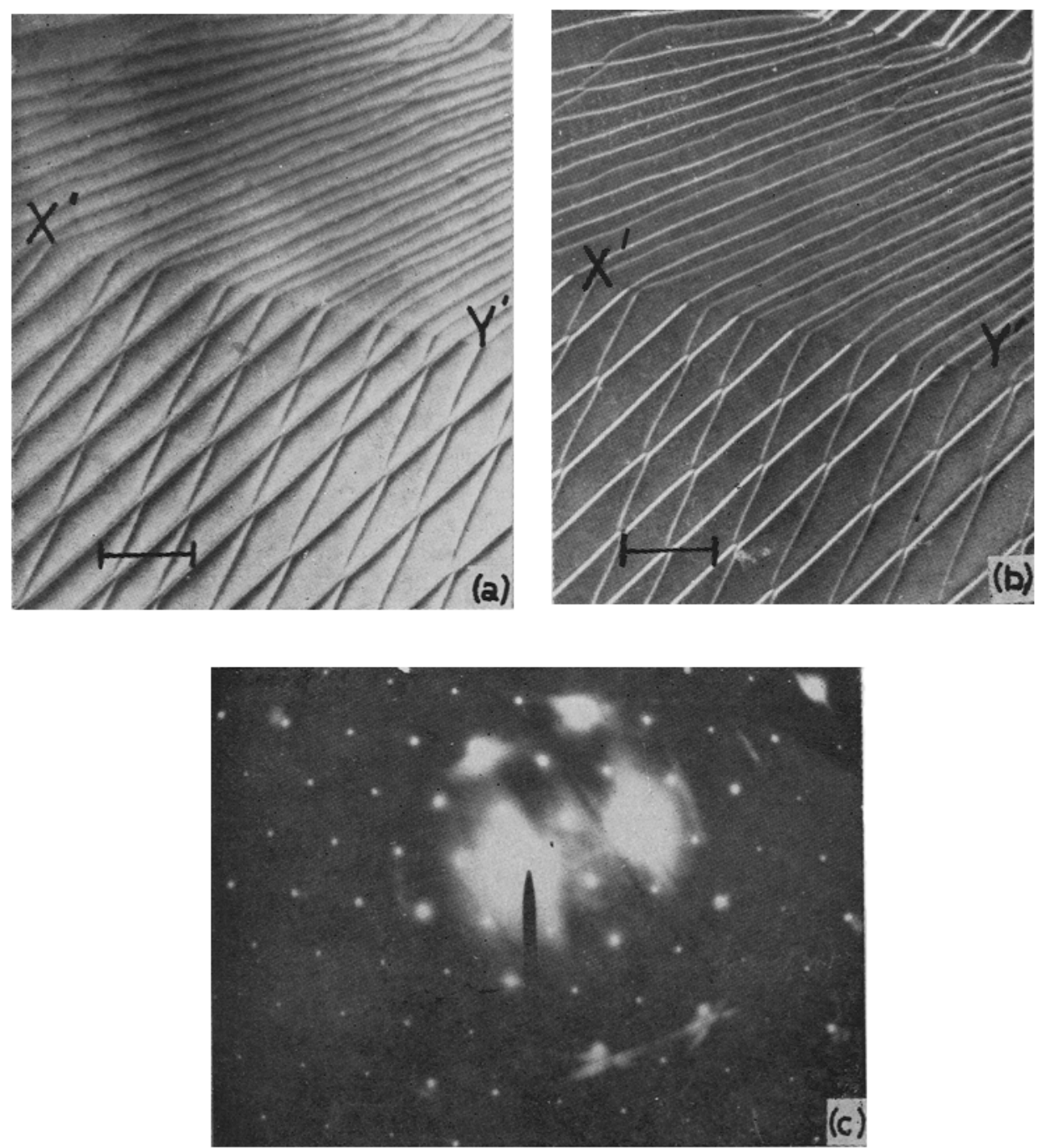

Figure 9a. Bright field picture of widening of two fold partials. Bar $=21 \mu \mathrm{m}$.

Figure 9b. Weak beam picture of figure $9 \mathrm{a}$. Bar $=21 \mu \mathrm{m}$.

Figure 9c. Selected area diffraction pattern of figure 9a. 
these ribbons cross the stacking fault triangles there is a change in the contrast. The fact that this change is only an electron optical effect is clearly revealed in the weak beam picture of the same region (figure 3 ), where no such thing is seen. The ribbons cross over the stacking fault triangles without forming any interaction with them. This is therefore quite clear that the ribbons are lying in a plane which is at a distance from the plane of the net. Also seen in the same photograph is the presence of a zigzag line $X Y$ crossing the stacking fault triangles, there is an interaction and the triangles shift in the direction $X Y$. This line can either be an isolated partial in the plane of the net or a twin boundary. The zigzag nature of the line and the fact that there is no change in the orientation of the triangles meeting the line, points to the first interpretation.

Figure $4 \mathrm{~b}$ is a weak beam picture of a network pattern as compared to figure $4 \mathrm{a}$, which is the bright field picture of the same region. Upon comparing these photographs carefully, we see that apart from the general increase in the sharpness of the dislocations, the weak beam picture also provides an easy interpretation of the photographs. In figure $4 b$, one can clearly see the formation of high energy extended and contracted nodes, a feature which is completely absent in the bright field pictures.

One more example of a network pattern formed from a family of ribbons is given in figure 5a. A comparison in figure $5 \mathrm{~b}$ (weak beam) with figure 5a shows that in the weak beam image there is a considerable increase in the resolution of the defects and it is noted that,

(i) The sharpness of the ribbons forming the network pattern in the weak beam image has vastly increased.

(ii) The node formation seen in figure 5a along the line $X_{1} X_{2}$ is not at all seen in figure $5 b$ and is thus an electron optical effect.

(iii) The interaction of the line $P Q$ with the ribbons forming the network pattern is clearly seen in figure $5 \mathrm{~b}$. Line $P Q$ intersects only with the ribbon along the $X_{1} X_{2}$ direction, while it has no effect on the ribbon in the $Y_{1} Y_{2}$ direction. At points marked $A$ and $B$ one can clearly note the shift in the ribbons where they interact with $P Q$.

During the course of the present work we came across a complicated network pattern formed from a family of three sets of ribbons. Figure 6 a shows a bright field picture of this type of network pattern, whereas figure $6 \mathrm{~b}$ is the weak beam picture of the same region. The formation of the pattern shown in figures $6 \mathrm{a}$ and $6 \mathrm{~b}$ can be clearly understood from the schematic diagram shown in figure 7. There is one set of ribbons marked by $A X$ which widen on reaching the boundary $P Q$. There is a second set of ribbons marked $B Y$. These ribbons have different widths in regions on both the sides of the line $P Q$. There is a third set of narrow ribbons $C Z$ which are only seen in the region left of $P Q$. Wherever ribbons $A X$ and $B Y$ cross each other they lead to the formation of high energy nodes (figures $6 \mathrm{a}$ and $6 \mathrm{~b}$ ) and also the points at which the ribbons $A X$ and $B Y$ cross $C Z$, a high energy node formation results. This feature can be very elegantly seen in the weak beam picture (figure $6 \mathrm{~b}$.)

Sometimes we come across a network pattern (figure 8 ) formed from a family of two sets ot two fold ribbons. In the left side of this figure, it is seen that generally there is no interaction between the two sets of ribbons except that there is a contrast change at the places where the two family of ribbons cross each other. There is one 
interesting feature of this photograph that partials forming the ribbons in the set II get widened when they reach the line marked as $X Y$ in the figure. The widened partials then interact with ribbons of set $I$ in the same manner as before.

This unique feature of widening of partials can be still more clearly seen in figures $9 \mathrm{a}$ and $9 \mathrm{~b}$. Here all the two fold ribbons upon reaching the boundary $X^{\prime} Y^{\prime}$ from the left get widened. A further interesting feature is the interaction between the widened ribbons on the right side of $X^{\prime} Y^{\prime}$. This interaction leads to the formation of a network pattern and whenever the ribbons cross, there is a twist at the crossing points. This twist can be attributed to a torque resulting from the strong forces which the dislocations exert locally on each other.

Figure $9 \mathrm{~b}$ is a weak beam picture of the region shown in figure 9a. It is seen that apart from the sharpness of the features in the weak beam picture, the interaction in the right side of $X^{\prime} Y^{\prime}$ is also very clearly seen in this picture.

The widening of ribbons upon reaching a boundary has been observed in past by Amelinckx et al (1963) in the case of compounds of graphite with bromine, iodine monochloride and ferric chloride. They have attributed this widening to the expansion of partials upon expansion of the interlayer spacing caused by the presence of ferric chloride. They have also noticed the widening of a dislocation ribbon by preferential diffusion of iodine monochloride along the ribbons.

In the present case $\mathrm{WS}_{2}$ crystals were grown by a direct vapour transport method where the incorporation of iodine or bromine which is always present in the chemically transported crystals is absent. Also the possibility of ferric chloride impurity is not at all feasible. However, a selected area diffraction pattern taken from the region showing the widening of ribbons (figure 9) is shown in figure 9c. Here one can easily see some faint spots refer either to some secondary phase of $\mathrm{WS}_{2}$ or some impurity which may lead to the observed widening of the ribbons.

\section{Conclusions}

Pictures showing network patterns in bright field have been compared with those taken by the weak beam technique. It is seen that the resolution in the weak beam pictures increases considerably, which helps in their interpretation.

Widening of ribbons on crossing a boundary has been observed quite often. A possible explanation for this phenomena has been produced.

\section{Acknowledgements}

The authors are grateful to Professor A R Patel for his keen interest in the work. KNR thanks UGC, New Delhi, for financial support. JVP is thankful to UGC for the award of Teacher Fellowship and is also thankful to the Principal and Management of Shree Jayendrapuri Arts and Science College, Broach for the grant of study leave. 


\section{References}

Agarwal M K and Bapu Joseph 1974 Indian J. Phys. 481129

Agarwal M K, Nagi Reddy K and Patel H B 1979a J. Crystal Growth 46139

Agarwal M K, Patel T C and Patel H B 1979b Proc. Indian Natl. Sci. Acad. 45392

Amelinckx S and Delavignette P 1962 Direct observation of Imperfections in crystals p. 295 (New York: Wiley)

Amelinckx S, Delavignette $\mathrm{P}$ and Heerscap M Paper presented at the 6th Biennal conference on carbon, Pittsburg (Pa) USA June 1963 pp. 17-21

Basinski Z S, Dove D B and Mooser E 1963 J. Appl. Phys. 34469

Basinskl Z S, Dove D B and Mooser E 1961 Helv. Phys. Acta 34373

Cockayne D J H, Ray I L F and Whelan M J 1969 Philos. Mag. 201265 